\title{
PROGRAMA BOLSA PERMANÊNCIA DA UFGD: O PERFIL SOCIOECONÔMICO DOS ALUNOS
}

\author{
PROGRAMA BOLSA PERMANENCIA DE UFGD: EL PERFIL SOCIOECONÓMICO \\ DE LOS ESTUDIANTES
}

\author{
BOLSA PERMANENCE PROGRAM UFGD: THE SOCIOECONOMIC PROFILE OF \\ STUDENTS
}

\author{
Adriana Carla Teixeira da Costa BRITO ${ }^{1}$ \\ Kellcia Rezende SOUZA ${ }^{2}$ \\ Vera Luci de ALMEIDA ${ }^{3}$
}

RESUMO: Toma-se como propósito estabelecer uma discussão sobre o perfil dos acadêmicos atendidos por ações afirmativas realizadas na Universidade Federal da Grande Dourados (UFGD). Tais ações nascem de políticas educacionais que se revelam como importantes ferramentas para o processo de democratização do acesso e, principalmente, a permanência ao ensino superior por extratos sociais menos abastados. Na presente investigação, o enfoque é o Programa Bolsa Permanência da UFGD e, entre os dados levantados, mostrou-se possível traçar um perfil do público-alvo atendido pela instituição. Defendemos que esse esforço em se estabelecer um perfil do acadêmico bolsista é um meio de contribuir para a otimização das ações afirmativas, isto é, consideramos que é a partir desse perfil que tais ações terão maiores condições de atender, de fato, as necessidades reais do seu público-alvo. São discussões feitas a luz de teóricos, tais como Schwartzman (2010) e Gomes (2018).

PALAVRAS-CHAVE: Democratização do Ensino Superior. Monitoramento e avaliação. Programa Bolsa Permanência.

RESUMEN: El propósito es establecer una discusión sobre el perfil de los estudiantes atendidos por acciones afirmativas realizadas en la Universidad Federal de Grande Dourados - UFGD. Tales acciones nacen de políticas educativas que se revelan como herramientas importantes para el proceso de democratización del acceso y, especialmente, la permanencia a la educación superior por extractos sociales menos ricos. En la presente investigación, el foco es el programa de bolsa permanencia de la UFGD y, entre los datos recogidos, fue posible rastrear un perfil del público objetivo al que asiste la institución. Argumentamos que este esfuerzo por establecer un perfil del erudito es un medio de

${ }^{1}$ Universidade Federal da Grande Dourados (UFGD), Dourados - MS - Brasil. Mestre em Administração Pública (Mestrado Profissional em Administração Pública em Rede - PROFIAP/UFGD. Pesquisadora na área de Políticas Públicas, Política de Assistência Estudantil. Orcid: https://orcid.org/0000-0002-3356-0456. E-mail: adrianacarlacosta@gmail.com

${ }^{2}$ Universidade Federal da Grande Dourados (UFGD), Dourados - MS - Brasil. Professora da Faculdade de Educação da UFGD. Orcid: https://orcid.org/0000-0002-8663-4615. E-mail: kellcia@ hotmail.com.

${ }^{3}$ Universidade Federal da Grande Dourados (UFGD), Dourados - MS - Brasil. Professora da Faculdade de Administração, Ciências Contábeis e Economia da UFGD. Orcid: https://orcid.org/0000-0003-1020-9169. Email: vera.vla@gmail.com. 
contribuir a la optimización de las acciones afirmativas, es decir, consideramos que es a partir de este perfil que tales acciones tendrán mayores condiciones para satisfacer, de hecho, las necesidades de su público objetivo. Estas son discusiones realizadas a la luz de teóricos como Schwartzman (2010) y Gomes (2018).

PALABRAS CLAVE: Democratización de la educación superior. Moniotreo y evaluación. Programa de becas de permanencia.

ABSTRACT: The purpose is to establish a discussion about the profile of the students attended by affirmative actions performed at the Federal University of Grande Dourados UFGD. Such actions are born from educational policies that are revealed as important tools for the democratization of access and, especially, the permanence to higher education by more wealthy social extracts. In the present investigation, the focus is the scholarship program of UFGD and, among the data collected, it was possible to trace a profile of the target audience attended by the institution. We argue that this effort to establish a profile of the scholar scholar is a means of contributing to the optimization of affirmative actions, that is, we consider that it is from this profile that such actions will have greater conditions to meet, in fact, the needs of your target audience. These are discussions made in the light of theorists such as Schwartzman (2010) and Gomes (2018).

KEYWORDS: Democratization of higher education. Monitoring and evaluation. Permanence grant program.

\section{Introdução}

Em tempos de discussões no cenário nacional acerca do futuro da Educação Superior, mostra-se imprescindível discutir as políticas que têm sido adotadas nas universidades públicas que visam a captação de recursos em benefício do acadêmico de baixa renda, a fim de que se garanta a sua permanência dentro da universidade e a sua boa formação. Ao lado disso, compreende-se como indispensável a ideia de questionar o modo como vem sendo feita a gestão dos recursos (em sentido de monitoramento e avaliação do processo), quando há, direcionados para este fim.

Dito isso, trazemos como proposta uma investigação sobre o perfil do acadêmico atendido pelo Programa Bolsa Permanência da Universidade Federal da Grande Dourados, implantado em 2007, que é uma das ações adotadas pela instituição como forma de aderir ao Programa Nacional de Assistência Estudantil (PNAES), instituído pela Portaria Normativa ${ }^{\circ}$ 39, de 12 de dezembro de 2007, e pelo Decreto $\mathrm{n}^{\circ}$ 7.234, de 19 de julho de 2010; e a forma como este recurso vem sendo gerenciado pela instituição, no período de 2014 a 2017.

Cabe salientar que os dados foram coletados na página oficial da UFGD, mediante buscas de documentos e informações sobre a Política de Assistência Estudantil na UFGD, tais 
como: Relatórios de Gestão, Relatório de Auditoria, Relatório de Avaliação dos Resultados da Gestão, Atos Administrativos (Instruções de Serviços, Editais e Resoluções) e, principalmente, procurou-se respaldo na busca de Regulamentos próprios do Programa Bolsa Permanência da UFGD. Todavia, para esta discussão, incluir-se-á uma proposta de leitura teórico crítica desses dados, sugerida por ambas as autoras, com base em suas respectivas experiências acadêmicas.

Dessa forma, destaca-se que para a obtenção desses dados, foram aplicados questionários junto aos acadêmicos de graduação da UFGD beneficiados pelo Programa Bolsa Permanência. E, nesta nova fase da pesquisa, propomos uma leitura desses resultados obtidos, com base em uma revisão da literatura que se tem sobre o assunto, a fim de se traçar o perfil desse aluno que é beneficiado pelo PNAES e, junto isto, trazemos uma proposta de monitoramento e avaliação para o Programa.

\section{Perfil socioeconômico dos estudantes assistidos pelo PNAES no Brasil}

Segundo Schwartzman (2010), a Universidade tem uma função democratizadora, esse se torna um dos principais motivos a nortear o processo de expansão do sistema educacional em todo o mundo. O direito à educação passou a ser considerado parte do conceito de cidadania, assim como o direito ao voto, à assistência social, ao salário-mínimo, às melhores condições de trabalho e aos direitos trabalhistas. O sistema educacional e, principalmente, a educação superior teriam por função oportunizar a todos, igual participação nos espaços sociais.

As ações afirmativas se definem como políticas públicas (e privadas) voltadas à concretização do princípio constitucional da igualdade material e à neutralização dos efeitos da discriminação racial, de gênero, de idade, de origem nacional e de compleição física. $\mathrm{Na}$ sua compreensão, a igualdade deixa de ser um princípio jurídico a ser respeitado por todos e passa a ser um objetivo constitucional a ser alcançado pelo Estado e pela sociedade. (GOMES, 2003. p. 21).

O FONAPRACE se dedicou ao levantamento de indicadores que pudessem demonstrar a importância da formulação de uma política voltada para essa demanda. Nas primeiras pesquisas realizadas pelo Fórum, procurou-se apresentar o perfil socioeconômico e cultural do estudante das IFES, destacando, sobretudo, a estratificação social, de modo a evidenciar a desigualdade entre os segmentos. Segundo consta no relatório da primeira pesquisa publicada, “[...] a preocupação básica dos órgãos de assistência é com aqueles 
alunos que estão abaixo do padrão médio das necessidades materiais, culturais e de serviços [...]". (FONAPRACE, 2012 p. 86).

Em relação à condição econômica dos estudantes, verificou-se que $44 \%$ pertenciam às classes C, D e E. Contudo, esse percentual é maior para as regiões Norte (69\%) e Nordeste (52\%). Tratando-se de renda familiar, $41 \%$ das famílias dos estudantes recebiam até 3 salários-mínimos, saltando os índices no Nordeste (50\%), Norte (63\%) e caindo no Sudeste (31\%) e Sul (32\%) (FONAPRACE, 2012). Na Tabela 1 é possível verificar a distribuição percentual dos estudantes segundo as regiões e as classes econômicas.

Tabela 1 - Distribuição percentual dos estudantes segundo região e classes econômicas -2010

\begin{tabular}{|c|c|c|c|c|c|c|c|c|c|}
\hline \multirow{2}{*}{$\begin{array}{c}\text { Região } \\
\text { Geográfica }\end{array}$} & \multicolumn{9}{|c|}{ Classes sociais } \\
\hline & A1 & A2 & B1 & B2 & $\mathrm{C1}$ & $\mathrm{C} 2$ & D & $\mathbf{E}$ & $\begin{array}{c}\mathbf{C}, \mathbf{D A} \\
\mathbf{E}\end{array}$ \\
\hline Nacional & 2,35 & 12,90 & 17,58 & 23,49 & 19,59 & 14,01 & 9,60 & 0,47 & 43,67 \\
\hline Nordeste & 2,20 & 9,96 & 14,57 & 21,26 & 20,11 & 17,10 & 14,00 & 0,81 & 52,02 \\
\hline Norte & 1,18 & 5,14 & 7,96 & 16,66 & 21,64 & 22,29 & 23,78 & 1,36 & 69,07 \\
\hline Sudeste & 2,22 & 16,11 & 21,68 & 25,56 & 18,41 & 11,27 & 4,67 & 0,07 & 34,42 \\
\hline Sul & 2,39 & 14,38 & 21,9 & 27,99 & 20,35 & 10,14 & 3,29 & 0,06 & 33,84 \\
\hline Centro-oeste & 4,30 & 17,76 & 18,27 & 23,74 & 18,27 & 23,74 & 18,09 & 10,74 & 6,75 \\
\hline
\end{tabular}

Fonte: Andifes/FONAPRACE

Nesse cenário, o FONAPRACE define os estudantes pertencentes às classes C, D e E como potenciais perfis de assistência estudantil, de forma a necessitar de algum tipo de apoio financeiro para a permanência e conclusão do curso, quer seja para custeio da alimentação, moradia, assistência à saúde, assistência psicológica, bolsas, dentre outros. Na Tabela 2, verifica-se a classe econômica dos estudantes das universidades federais em relação às pesquisas do FONAPRACE dos anos de 1996/07, 2003/04 e 2010.

Tabela 2 - Classes econômicas dos estudantes das universidades federais por anos de pesquisas

\begin{tabular}{c|c|c|c}
\multirow{2}{*}{ Classe Econômica } & \multicolumn{3}{|c}{ Região Geográfica } \\
\cline { 2 - 4 } & Pesquisa & Pesquisa & Pesquisa 2010 \\
\hline A & $1996 / 97$ & $\mathbf{2 0 0 3 / 0 4}$ & $\mathbf{1 5 , 6 \%}$ \\
\hline B & $\mathbf{1 2 , 6 \%}$ & $\mathbf{4 1 , 5 \%}$ & $\mathbf{4 1 , 1 \%}$ \\
\hline C & $\mathbf{3 0 , 5 \%}$ & $\mathbf{3 0 , 9 \%}$ & $\mathbf{3 3 , 6 \%}$ \\
\hline D & $10,5 \%$ & $\mathbf{1 1 , 1 \%}$ & $\mathbf{9 , 6 \%}$ \\
\hline E & $\mathbf{3 , 3 \%}$ & $\mathbf{0 , 8} \%$ & $\mathbf{0 , 5 \%}$ \\
\hline C+D+E & $\mathbf{4 4 , 3 \%}$ & $\mathbf{4 2 , 8 \%}$ & $\mathbf{4 3 , 7 \%}$
\end{tabular}

Fonte: Andifes/FONAPRACE 
Reitera-se, nesse sentido, que as políticas de assistência estudantil são necessárias, uma vez que os estudantes das Universidades Federais não são, em sua maioria, os mais ricos, e sim os de classes socioeconomicamente vulneráveis (FONAPRACE, 2012). Inclusive, o percentual daqueles que necessitam de assistência estudantil estão em sua grande maioria na região Nordeste, Centro-Oeste e, em maior concentração, na região Norte. O grupo de estudantes de faixa etária de até 24 anos representa $74,53 \%$ dos estudantes de graduação das IFES brasileiras, o que demonstra serem esses estudantes majoritariamente jovens. Segundo o relatório da pesquisa de 2010, a maioria dos estudantes das universidades federais são no sexo feminino, em torno de 53,0\%. Além disso, aponta-se que a região com a maior concentração é do Norte, com 57,0\% de seus estudantes sendo do sexo feminino (FONAPRACE, 2012). Conforme pode ser observado na Tabela 3 , a seguir.

Tabela 3 - Distribuição percentual dos estudantes por sexo, idade e região - 2010

\begin{tabular}{|c|c|c|c|c|}
\hline \multirow{3}{*}{ Norte } & \multirow{3}{*}{$\begin{array}{l}\text { Até } 17 \text { anos } \\
\text { De } 18 \text { a } 24 \text { anos } \\
25 \text { anos ou mais }\end{array}$} & $0,59 \%$ & $1,09 \%$ & $0,88 \%$ \\
\hline & & $69,73 \%$ & $68,88 \%$ & $69,23 \%$ \\
\hline & & $29,68 \%$ & $30,04 \%$ & $29,89 \%$ \\
\hline \multirow{3}{*}{ Sudeste } & \multirow{3}{*}{$\begin{array}{l}\text { Até } 17 \text { anos } \\
\text { De } 18 \text { a } 24 \text { anos } \\
25 \text { anos ou mais }\end{array}$} & $0,16 \%$ & $0,31 \%$ & $0,24 \%$ \\
\hline & & $74,00 \%$ & $80,61 \%$ & $77,58 \%$ \\
\hline & & $25,84 \%$ & $19,09 \%$ & $22,18 \%$ \\
\hline \multirow{3}{*}{ Sul } & \multirow{3}{*}{$\begin{array}{l}\text { Até } 17 \text { anos } \\
\text { De } 18 \text { a } 24 \text { anos } \\
25 \text { anos ou mais }\end{array}$} & $1,73 \%$ & $1,09 \%$ & $0,91 \%$ \\
\hline & & $67,65 \%$ & $74,05 \%$ & $70,88 \%$ \\
\hline & & $31,62 \%$ & $24,86 \%$ & $28,21 \%$ \\
\hline \multirow{3}{*}{ Centro-Oeste } & \multirow{3}{*}{$\begin{array}{l}\text { Até } 17 \text { anos } \\
\text { De } 18 \text { a } 24 \text { anos } \\
25 \text { anos ou mais }\end{array}$} & $2,24 \%$ & $2,41 \%$ & $2,33 \%$ \\
\hline & & $72,45 \%$ & $74,24 \%$ & $73,40 \%$ \\
\hline & & $25,31 \%$ & $23,35 \%$ & $24,27 \%$ \\
\hline
\end{tabular}

\section{Fonte: Andifes/FONAPRACE}

Quanto à idade, $75,74 \%$ do total das estudantes do sexo feminino se encontra na faixa etária de 18 a 24 anos e a maior concentração encontrada na pesquisa é na região sudeste, onde $80,61 \%$ são do sexo feminino e $74 \%$ do sexo masculino (FONAPRACE, 2012). O cenário atual da Política de Assistência Estudantil nas universidades públicas e a relevância do PNAES na articulação com o ensino, pesquisa e extensão - com foco principal na permanência dos estudantes no ensino superior público - possibilitam a compreensão do contexto político e legal em que se insere o programa Bolsa Permanência da Universidade Federal da Grande Dourados, objeto deste estudo. 


\section{O perfil do bolsista do programa bolsa permanência da UFGD}

A Universidade Federal da Grande Dourados (UFGD) foi criada no ano de 2005, conforme a Lei $\mathrm{n}^{\mathrm{0}} 11.153$, de 29 de julho de 2005 e implantada, sob a tutoria da UFG, no ano de 2006. E o PNAES da UFGD iniciou com o processo de regulamentação da concessão de benefícios assistências aos estudantes em 2011, pela Resolução nº 173/2011, aprovada pelo Conselho Universitário. O Programa promove diferentes ações, a exemplo da concessão de auxílios permanência e alimentação, o apoio pedagógico e psicossocial, além de ações e práticas desportivas.

O Programa Bolsa Permanência (PBP), que é o foco dessa discussão, está inserido na política de Assistência Estudantil da UFGD. A escolha deste Programa por nós se justifica na medida em que se trata do maior percentual utilizado do recurso do PNAES, como também por estar diretamente ligada a permanência dos alunos com vulnerabilidade socioeconômica na instituição. O Programa em questão proporciona um aporte financeiro, por meio de bolsas, a acadêmicos selecionados por critérios de avaliação socioeconômica e tem por objetivo viabilizar a permanência do aluno na universidade.

Para a classificação dos estudantes como perfil, a Pró - reitoria utiliza uma metodologia a combinação da Análise Socioeconômica e o Índice de Classificação (IC), o qual consiste no cálculo da renda per capita familiar, atrelado a índices redutores ou de incentivo. O cálculo do IC é formado por variáveis que permitem priorizar os estudantes que apresentam aspectos que podem ser indicadores de vulnerabilidade social e econômica. Com isso, já no ano de 2014, o PBP disponibilizava 550 bolsas e, em 2017, alcançou o total de 1.543 bolsas, segundo editais disponíveis no site da instituição ${ }^{4}$. Para concessão de bolsas os editais adotam critérios como:

[...] realizar a Avaliação Socioeconômica de que trata o Regulamento do Processo de Avaliação Socioeconômica, e ser classificado no Perfil I; estar regularmente matriculado com carga horária mínima de 432 horas/aula no semestre para os cursos integrais de graduação e carga horária mínima de 288 horas/aula, no semestre para os demais cursos presenciais de graduação da UFGD; entregar o termo de compromisso assinado na PROAE; além de atender as demais ponderações do Regulamento de Assistência Estudantil: Bolsa Permanência (UFGD, 2017).

${ }^{4}$ Edital PROAE/UFGD no 17, de 24 de junho de 2014; Edital PROAE/UFGD no 45 de 13 de novembro de 2015; Edital PROAE/UFGD no 13 de 27 de abril de 2016; edital PROAE/UFGD/Vagas Remanescentes $n^{\circ} 30$ de 10 de junho de 2016; Edital PROAE/UFGD n 10 de 03 de fevereiro de 2017; Edital PROAE/UFGD n 141 de 31 de julho de 2017 e Edital PROAE/UFGD nº 180 de 18 de dezembro de 2017. 
E com isso, para analisarmos o perfil do bolsista tomamos como metodologia, em um primeiro momento, o levantamento dos documentos produzidos pela instituição, no que se refere à distribuição de bolsas. Mediante os relatórios de pagamentos e de indicadores da Pró Reitoria de Assuntos Estudantis, a PROAE, foram extraídos dados que viessem a colaborara para a feitura de um processo de avaliação e de monitoramento do Programa Bolsa Permanência da UFGD.

Dessa forma, conforme Gráfico 1, procuramos evidenciar o número de estudantes por sexo, no qual se percebe um maior predomínio dos beneficiários do sexo feminino. Ou seja, dos anos analisados, o percentual de mulheres que foram contempladas no processo sempre foi igual ou superior a $60 \%$ do total de bolsistas, conforme pode ser observado a seguir:

Gráfico 1 - Beneficiários, por sexo de 2014 a 2017

\begin{tabular}{|c|c|c|}
\hline EDITAL REMANESCENTE 2017 № 180 & 0,40397351 & 0,59602649 \\
\hline EDITAL REMANESCENTE 2017 № 141 & 0,349206349 & 0,650793651 \\
\hline EDITAL 2017 № 10 & 0,37388724 & 0,62611276 \\
\hline EDITAL REMANESCENTE 2016 № 30 & 0,307692308 & 0,692307692 \\
\hline EDITAL 2016 № 13 & 0,350671141 & 0,649328859 \\
\hline EDITAL 2015 № 45 - 1a CHAMADA & 0,358974359 & 0,641025641 \\
\hline EDITAL 2014 № 17 - 1a CHAMADA & 0,323636364 & 0,676363636 \\
\hline
\end{tabular}

Fonte: Gráfico elaborado pela autora com base nos Editais de divulgação PROAE/UFGD (2018)

A partir desses dados, há a possibilidade de visualizar alterações substanciais no processo de ensino do país, isto é, é possível perceber um aumento significativo da presença feminina no espaço universitário. Embora ainda se observe que um dos maiores desafios das mulheres atualmente seja poder adentrar em cursos que continuam sendo predominantemente masculinos e conseguir permanecer na universidade.

Na sequência, o Gráfico 2 apresenta a distribuição dos beneficiários do Programa Bolsa Permanência da UFGD por faculdade, durante os anos de 2014 a 2017, período de divulgação dos relatórios de indicadores da PROAE: 
Gráfico 2 - Beneficiários, do Programa Bolsa Permanência da UFGD, por faculdade, durante os anos de 2014 a 2017

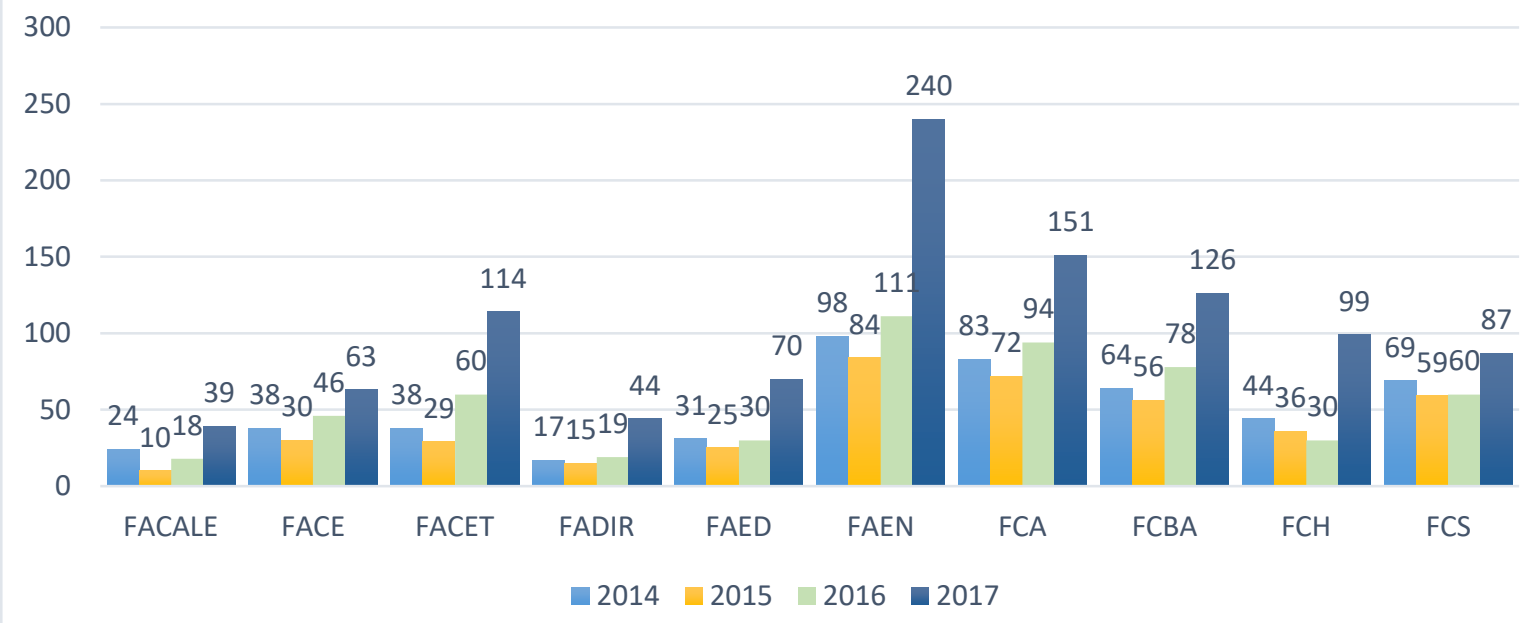

Fonte: Gráfico elaborado pela autora com base nos Relatórios de pagamentos e Relatórios de Indicadores PROAE/UFGD. Org.: DIPLAN/COPLAN (2014 a 2016).

Durantes esses anos, foram beneficiados com o Programa Bolsa Permanência da UFGD 2.501 alunos, conforme demonstrado no Gráfico 2. Observa-se que a faculdade com maior quantitativo de alunos beneficiados pelo Programa Bolsa Permanência é a Faculdade de Engenharia (FAEN), com um total de 533 bolsistas, e que logo após está a Faculdade de Ciências Agrárias (FCA), com 400 beneficiários. Seguidas das Faculdades de Ciências Biológicas e Ambientais (FCBA), com 324 estudantes e a Faculdade de Ciências Exatas e Tecnologia (FACET), com 241 alunos bolsistas.

Segundo Gross, Hossler e Ziskin (2007), os estudantes matriculados em cursos de quatro anos com o apoio financeiro fornecido pelas próprias instituições de ensino tem um efeito positivo e estatisticamente significante, apesar de moderado, sobre a permanência dos alunos. Dentre os motivos encontrados no estudo, ao receber o benefício, os indivíduos têm uma menor necessidade de trabalhar, o que permite um maior engajamento na vida universitária.

De forma geral, considera-se a hipótese na qual estudantes que contribuem com o próprio sustento e da família estão matriculados predominantemente em cursos noturnos, uma vez justificado o fato de o aluno ter um emprego o qual não o permita estudar em período integral. Entretanto, a partir deste estudo, pôde-se compreender que muitos alunos do bacharelado vêm de uma situação socioeconômica mais vulnerável, sendo os maiores recebedores de auxílios do governo para se sustentar durante a realização do curso superior.

Nesse sentido, o monitoramento mais uma vez se faz necessário devido à prioridade de atendimento dos estudantes em situação de vulnerabilidade socioeconômica, quanto ao 
critério de seleção e concessão de benefício. Nota-se que as faculdades com o maior percentual de recebimento de bolsas são as de cursos integrais, nos quais os alunos não conseguem adentrar ao mercado de trabalho em virtude dos horários de estudos.

Para Mello (2007), variáveis como renda familiar, escolaridade paterna e ocupação do chefe da família influenciam não somente na probabilidade de ingresso no ensino superior, mas também nos riscos de evasão. Dessa forma, esses fatores estão diretamente relacionados com a busca pelos programas de permanência.

A partir do ano de 2014 houve um aumento significativo na quantidade de bolsas permanência concedidas aos alunos dos cursos integrais. Para o caso da amostra dessa pesquisa, 352 estudantes dos cursos integrais foram atendidos em 2014 e, já em 2017, esse número subiu para 718 beneficiários.

Dessa forma, procura-se avaliar se o aumento na quantidade de bolsas foi acompanhado pela diminuição da evasão entre os beneficiários. $\mathrm{O}$ auxílio financeiro dado pelas universidades, segundo Gross, Hosler e Zinski (2007), tem um impacto positivo, porém, moderado, na persistência do estudante. Araújo e Leite (2014) corroboram que os estudantes que receberam o auxílio também abandonavam menos os cursos.

\section{Considerações finais}

Como se pode observar, podemos fazer a leitura dos dados resultantes da pesquisa feita, da seguinte forma: o perfil predominante do acadêmico bolsista do Programa Bolsa Permanência, instituído pela Universidade Federal da Grande Dourados, no período de 2014 a 2018, é proveniente da classe social C, D ou E; é um perfil feminino e com predominância nas ciências exatas e biológica.

A partir de um perfil criado para o acadêmico bolsista é possível partir para uma segunda fase da pesquisa, isto é, a análise das reais necessidades deste aluno que transcende o espaço da sala de aula (tais como alimentação, transporte, moradia, entre outros), mas que, como a história da educação vem nos apresentando, impactam diretamente no desenvolvimento da sua formação acadêmica e, por conseguinte, na sua permanência no contexto de ensino.

Além disso, também compreendemos que é a partir deste perfil e do real atendimento das suas necessidades que há uma otimização dos esforços em prol desta conquista. Isto é, uma vez tendo em mãos o perfil desse aluno e da sua real necessidade, o processo de destinação de recursos passa a ser mais preciso e, por conseguinte, é feita uma melhor 
distribuição de recursos entre as ações afirmativas do programa - sendo esta também a nossa preocupação em um desdobramento desta pesquisa.

\section{REFERÊNCIAS}

ARAÚJO, J. C. F. de; LEITE, L. S. Avaliação da política de apoio ao estudante desenvolvida pela UNIRIO: o Projeto de Bolsa Permanência. Ensaio: Avaliação e Políticas Públicas em Educação. Rio de Janeiro, v. 22, n. 84, p. 777-806, jul./set. 2014. Disponíveis em: http://www.scielo.br/pdf/ensaio/v22n84/a09v22n84.pdf. Acesso em: 17 nov. 2017.

BRASIL. Decreto ${ }^{\circ} 7.234$ de 19 de julho de 2010. Dispõe sobre o Programa Nacional de Assistência Estudantil - PNAES. Brasília, 2010. Acesso em: 20 set. 2017.

FONAPRACE. Revista Comemorativa 25 Anos: histórias, memórias e múltiplos olhares / Organizado pelo Fórum Nacional de Pró-reitores de Assuntos Comunitários e Estudantis, coordenação, ANDIFES. - UFU, PROEX: 2012. Acesso em 03 jun. 2017.

GOMES, J. Direito, Sociedade Civil e Minorias no Brasil da Virada do Milênio. In: DORA, D. D. (Org.). Direito e Mudança Social. Rio de Janeiro: Renovar, 2003. Acesso em: 15 ago. 2018.

GROSS, J.; HOSSLER, D.; ZISKIN, M. Institutional Aid and Student Persistence: An Analysis is of the Effects of Institutional Financial Aid at Public Four-Year Institutions. NASFAA Journal of Student Financial Aid, v. 37, n. 1, 2007. Acesso em: 25 ago. 2017.

MELLO, J. C. R. S. Desigualdades sociais e acesso seletivo ao ensino superior no Brasil no período 1994-2001. Revista Electrónica Iberoamericana sobre Calidad, Eficacia y Cambio en Educación, Madrid. Espana, v. 5, n. 2, p. 69-83, 2007. Disponível em: http://www.rinace.net/arts/vol5num2e/art6.pdf. Acesso em: 27 maio 2018.

SCHWARTZMAN, S. ENEM: o que realmente importa. Belo Horizonte, 2010. Disponível em: http://www.schwartzman.org.br/sitesimon/?p=1907\&lang=pt-br . Acesso em: 31 mar. 2018.

UNIVERSIDADE FEDERAL DA GRANDE DOURADOS (UFGD). Edital PROAE/UFGD no 17, de 24 de junho de 2014.

UNIVERSIDADE FEDERAL DA GRANDE DOURADOS (UFGD). Edital PROAE/UFGD $n^{0} 45$ de 13 de novembro de 2015.

UNIVERSIDADE FEDERAL DA GRANDE DOURADOS (UFGD). Edital PROAE/UFGD n $^{0} 13$ de 27 de abril de 2016.

UNIVERSIDADE FEDERAL DA GRANDE DOURADOS (UFGD). Edital PROAE/UFGD/Vagas Remanescentes $n^{\circ} 30$ de 10 de junho de 2016.

UNIVERSIDADE FEDERAL DA GRANDE DOURADOS (UFGD). Edital PROAE/UFGD $n^{\circ} 10$ de 03 de fevereiro de 2017 
UNIVERSIDADE FEDERAL DA GRANDE DOURADOS (UFGD). Edital PROAE/UFGD $n^{\circ} 141$ de 31 de julho de 2017.

UNIVERSIDADE FEDERAL DA GRANDE DOURADOS (UFGD). Edital PROAE/UFGD $n^{\circ} 180$ de 18 de dezembro de 2017.

\section{Como citar este artigo}

BRITO, Adriana Carla Teixeira da Costa; SOUZA, Kellcia Rezende; ALMEIDA, Vera Luci de. Programa Bolsa Permanência da UFGD: o perfil socioeconômico dos alunos. Doxa: Rev. Bras. Psico. e Educ., Araraquara, v. 21, n. 1, p. 17-27, jan./jun. 2019. e-ISSN: 2594-8385. DOI: $10.30715 /$ doxa.v21i1.12978

Submetido em: 01/11/2018

Revisões requeridas: 10/12/2019

Aprovado em: 16/12/2019

Publicado em: 01/02/2019 